\title{
Assessment of Integrated Sub-Micron Polysilicon Fuses for Low Voltage CMOS Applications
}

Jake Schaper, Theresa Hopson*, Alex VanVianen

Motorola Semiconductor Products Sector, Arizona Product Analysis Lab

2100 E. Elliot Road, Tempe, AZ 85284

*Motorola Physical Sciences Research Labs, 7700 S. River Parkwy, Tempe, AZ 85284

Sacrificial fuses are used in many applications where proprietary internal programming of integrated circuits is required. After programming, a sacrificial fuse is intentionally pulsed with enough energy to effectively open the program path. The open path prevents subsequent unauthorized interrogation of the internal program. This paper presents an evaluation of the physical properties of the pulsed fuse and the characteristics of the resulting agglomerated region that is created during the pulse.

An integrated polysilicon fuse, Figure 1, is defined during the fabrication of the chosen circuit to be protected. The fuse is a two-layer structure comprised of a cobalt salicide layer deposited on a polysilicon layer with the dimensions of 2 microns long by 0.18 microns wide. The entire fuse structure is deposited on an oxide film on a silicon substrate. The interconnect scheme incorporates tungsten plugs between an aluminum/copper layer and the underlying cobalt/salicide. Typically, after the internal circuitry is programmed, an electrical pulse of controlled voltage and duration is applied to effectively open the fuse, thereby inhibiting later interrogation of the circuit. Fuses that were exposed to various voltages and durations were submitted for documentation of the resulting agglomerated regions.

After deprocessing the circuits to the oxide level above the fuses, a cross-section was generated using focused ion beam (FIB) milling. Figure 2 shows a typical cross-section of a pulsed fuse. Localized disruption of the surrounding oxide layers was not detrimental to circuit performance and there was no evidence of thermally induced cracking. SEM and TEM images showed tungsten along the fuse plane. The effect of tungsten in the fuse region, and the behavior of the $\mathrm{CoSi}_{2}$ layer are evaluated with thermal imaging.

1. K. Lim, et al., 2001 Symposium on VLSI Circuits Digest of Technical Papers, (2001) pp. 33-34.

2. A. Kalnitsky et al., IEEE IEDM, (1999) 765.

3. Handbook of Chemistry and Physics, CRC Press, 69th Ed. 1988-1989. 


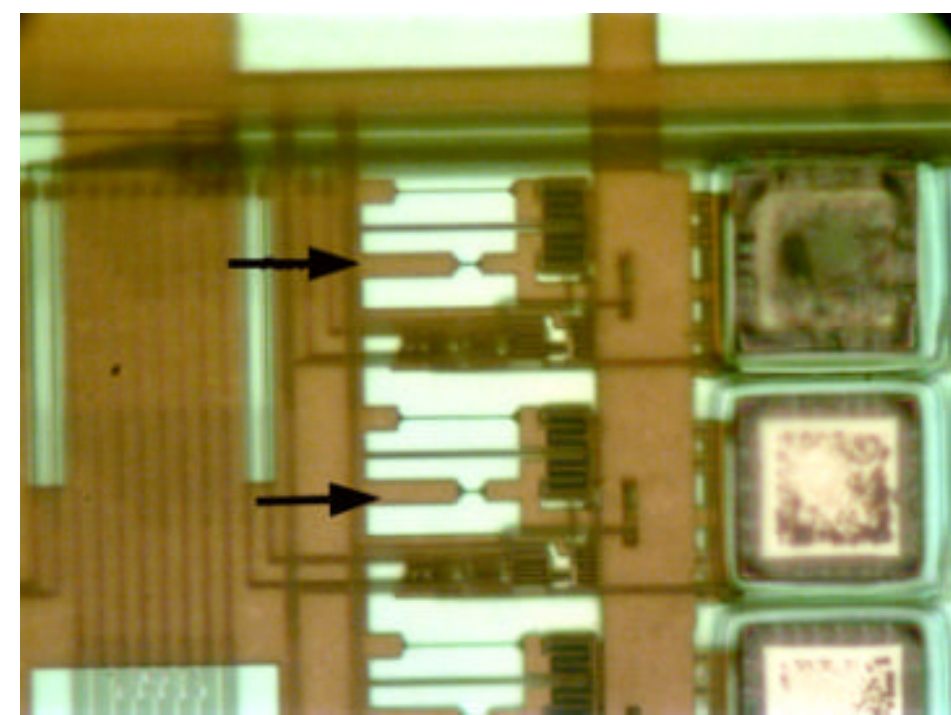

FIGURE 1. Confocal image of two fuses, as indicated by arrows.

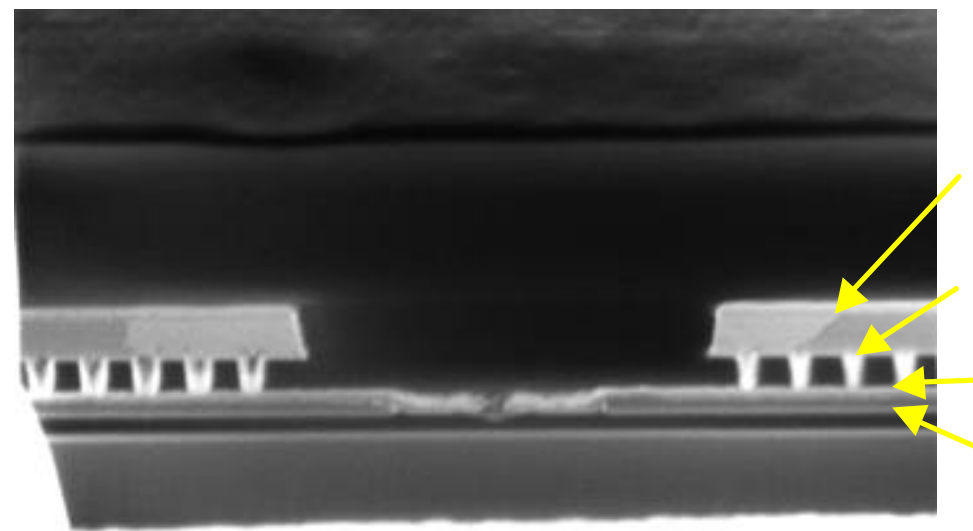

Aluminum/Copper

Tungsten Interconnect

Cobalt Salicide

Polysilicon

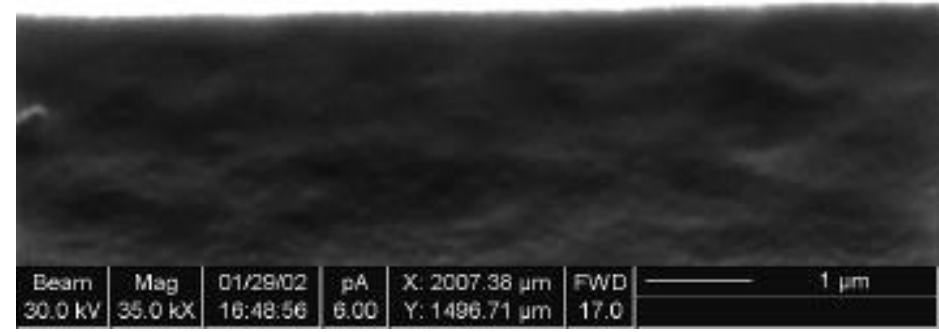

FIGURE 2. FIB-generated cross-section showing the components of a pulsed fuse and related interconnnecting region. 